\title{
Resampling and Data Augmentation For Equines' Behaviour Classification Based on Wearable Sensor Accelerometer Data Using a Convolutional Neural Network
}

\begin{abstract}
Monitoring horses' behaviors through sensors can yield important information about their health and welfare. Sampling frequency majorly affects the classification accuracy in activity recognition and energy needs for the sensor. The aim of this study was to evaluate the effect of sampling rate reduction of a tri-axial accelerometer on the recognition accuracy by resampling a $50 \mathrm{~Hz}$ experimental dataset to four lower sampling rates $(5 \mathrm{~Hz}, 10 \mathrm{~Hz}, 12.5 \mathrm{~Hz}$ and $25 \mathrm{~Hz}$ ). Also, in this work we investigate the 'reality gap' that incorporates changes in the data that are primarily characterized as sensor rotations or measurement noise through various data augmentation techniques such as rotation and jittering. Finally, another factor influencing activity recognition are the subjects themselves and therefore the model is evaluated on different horse types. A deep learning-based approach for activity detection of equines is proposed to automatically classify 2238 manually annotated $2 \mathrm{~s}$ samples tri-axial accelerometer leg data data of seven different activities performed by six different subjects. The raw data are preprocessed and fed into a convolutional neural network (CNN) from which features are extracted automatically by using strong computing capabilities. Furthermore, the neural network was intentionally designed to minimize running time, enabling us to imagine the future use of the built model in embedded constrained devices.

The complexity of these automatic learning techniques can be decreased while achieving high accuracies using ten-fold-cross validation using a computationally less intensive received signal length data $\mathbf{( 9 9 . 3 2 \%}$ at $5 \mathrm{~Hz}$ vs $99.74 \%$ at $25 \mathrm{~Hz}$ ). This indicates that sampling at $5 \mathrm{~Hz}$ with a $2 \mathrm{~s}$ window will offer advantages for activity surveillance thanks to decreased energy requirements, since validation time decreases 16-fold (784 microseconds at $50 \mathrm{~Hz}$ to 48 microseconds at $5 \mathrm{~Hz}$ ). Moreover, in this work we show that rotating the training or validation signal with 10 degrees over the $X, Y$ and $Z$-axis increases the generalization capabilities of our model $(99.61 \%$ vs $99.93 \%$ ) while adding small amounts of noise (smaller than 0.3 standard deviation (STD)) does not decrease the classification accuracy under $99 \%$. Finally, the performance and ability of the model to generalize is validated on data from unseen horses at the cost of only $4.1 \%$ and $2.45 \%$ reduction in accuracy when validated on a pony and a lame horse, respectively.
\end{abstract}

Index Terms-Data augmentation, equines, accelerometer, convolutional neural networks, behaviour classification, resampling, internet-ofanimals.

\section{INTRODUCTION}

With innovative biosensors becoming widely available, different applications such as disease identification, health monitoring and detection of reproductive cycles could be used for an improved livestock management [1]. This trend also entered the equestrian world, since monitoring horses' behaviour can yield important information about their health and welfare [2]. Different technologies have been designed to detect various parameters such as activity, elevation, heart rate, etc., from which conclusions can be drawn about the horses' behaviour [3], [4], [5]. Wearable accelerometers in particular have been tested for the determination of gaits by defining distinct ranges of acceleration values for stand, walk, trot and canter, but not yet for other behaviors such as rolling, pawing and flank watching [4]. Also, researchers have suggested the use of various machine learning tools (K-nearest neighbours, Naive Bayes, Support vector machine) to classify lying, standing, and feeding behaviour of dairy cows from accelerometer data more accurately [6]. A disadvantage of the proposed methods is that feature extraction is still necessary, while
Convolutional Neural Networks (CNNs) have the advantage of automatic feature extraction with only limited computational complexity [7]. Deep learning-based classifiers can learn features and achieve better accuracy [8]. Studies have shown that deep learning models are capable of learning and discriminating between human activities such as e.g, sitting, walking, climbing upstairs, walking downstairs or falling but to the authors' knowledge they have not yet been applied to detect equine activities [9]. Further, since configuration parameters such as accelerometer sampling rate and sensor positioning can influence activity identification performance, it is important to train a deep neural network that generalizes well to new data [10]. Therefore, data augmentation techniques are used to construct distinct training and test sets to control the overfitting of data during training, a considerable drawback endangering generalization of the model [11]. It is demonstrated that significant generalization gains in the learning process are attained by randomly generating augmented training data using geometric transformations such as rotation, permutation, scaling and magnitude-warping [12], [13] and [14]. The application of small amounts of input noise (jitter) to the training data can aid to model generalization and increase model robustness, as it is a way to model multiplicative and additive sensor noise [15]. Deviations in sensor positioning are simulated by applying rotations to existing data. This has been shown to greatly enhance the classifier's generalization capabilities, as the CNN is forced to learn feature representations that are invariant to the accelerometer orientation [14].

This paper aims to investigate the effect of lower sampling rates, sensor rotations, measurement noise and type of horse on the algorithm recognition accuracy. The remainder of the paper is organized as follows. Section 2 deals with the methodology and the proposed deep learning model. Section 3 describes the findings of the experimentally validated model. Ultimately, in Section 4, conclusions are drawn.

\section{MATERIALS AND METHOD}

\section{A. Animals and study site}

Measurements were carried out on a horse farm in Zutendaal, Belgium with six adult horses of distinct breeds, between November 2018 and April 2019. Detailed information about the selected subjects is presented in Table I. Owners or familiar riders carried out the exercising for data recording at a local practice arena with a size of $25 \mathrm{~m} \mathrm{x} 38 \mathrm{~m}$ and a track surface of sand mixed with GEOPAT polyflakes. A minority of the data is collected on a meadow and a hard (concrete) underground.

\section{B. Data collection procedure}

Sensor data are collected utilizing Axivity AX3 accelerometers (Axivity Ltd, Newcastle, United Kingdom) with a dimension of $23 \times 32.5 \times 7.6 \mathrm{~mm}$ and a weight of $11 \mathrm{~g}$. These $\log$ data with configurable sampling rates ranging from $12.5 \mathrm{~Hz}$ to $3200 \mathrm{~Hz}$ and are powered by a $150 \mathrm{mAh}$ lithium-polymer battery which is rechargeable via USB connection. Acceleration is measurable on $\mathrm{x}-, \mathrm{y}-$, $\mathrm{z}$-axes with a maximum sensitivity of $\pm 16 g\left[g=m / s^{2}\right]$. The AX3 OMGUI 
TABLE I

PARTICIPATING HORSES WITH BREED CLASS, HEIGHT AT WITHERS, GENDER, AGE, STATE AND TYPE OF SHOEING.

\begin{tabular}{lllllll}
\hline No. & $\begin{array}{l}\text { Breed } \\
\text { class }\end{array}$ & $\begin{array}{l}\text { Height } \\
\text { at } \\
\text { withers } \\
\text { (cm) }\end{array}$ & Gender & Age & State & Shoeing \\
\hline 1 & Warmblood & 172 & Mare & 7 & Healthy & Barefoot \\
2 & Warmblood & 167 & Gelding & 11 & Healthy & Barefoot \\
3 & Warmblood & 181 & Mare & 17 & Lame & Barefoot \\
4 & Warmblood & 168 & Mare & 19 & Healthy & Barefoot \\
5 & Friesian & 159 & Mare & 12 & Healthy & Shoed \\
6 & Pony & 116 & Gelding & 15 & Healthy & Barefoot \\
\hline
\end{tabular}

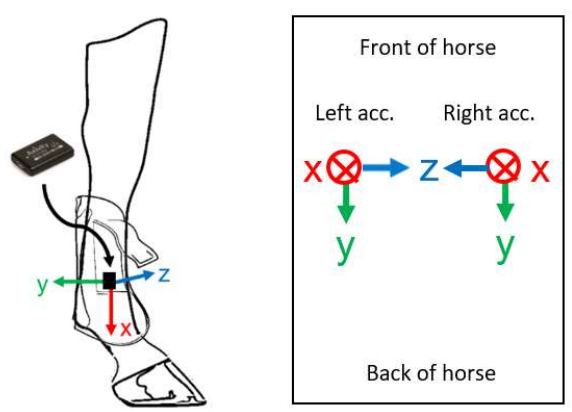

Fig. 1. Left: Position and orientation (X, Y, and $\mathrm{Z}$ axes) of the right accelerometer. Right: top view of orientation of left and right accelerometer.

Configuration and Analysis Tool, an open source application, is used to set up and configure the AX3 sensors for data recording. Data is registered on an embedded $512 \mathrm{MB}$ memory.

The accelerometers were attached to six horses at the two front legs at the lateral side of the tendon boot securely fastened with VELCRO stick on circles to the tendon boot, with minimal room for vibration, slip or twist; to ensure that only the horses' motions are captured. All devices were fixed using the orientation illustrated in Figure 1 with the three colored axes indicating the orientation of the accelerometer axes.

In this study, the device sensors were set to collect data from all the considered activities as listed in Table II with their descriptive definitions. Observations of the activities of the horses were made with video recordings concurrently with the collection of sensor data. After recording data was transferred to a computer via USB connection and stored in a Continuous Wave Accelerometer format. All the data is labelled by a trained observer based on the video recordings using ELAN, which is a specialized video annotation software, since it is difficult to use direct observation in conjunction with training of the horse [16]. In Table III the number of $2 \mathrm{~s}$ instances for all horses and the class proportions are presented. As can be concluded from this table, the activity walk is the most present with a class proportion of $49.6 \%$ and paw is the least present behavior in the dataset with only 20 instances.

\section{Machine learning model}

A multilayer convolutional network as shown in Figure 2 is used with two convolutional layers, the first followed by a max-pooling layer. The second convolutional layer is followed by two fully connected layers from which the output of the last fully-connected layer is fed into a 7-way softmax layer that generates a distribution over the seven class labels: stand, walk, trot, canter, roll, paw, flank watching.
TABLE II

DESCRIPTION OF THE OBSERVED ACTIVITIES [17]

\begin{tabular}{ll}
\hline $\begin{array}{l}\text { Observed } \\
\text { activities }\end{array}$ & Description \\
\hline Stand & $\begin{array}{l}\text { The horse stands at least three legs without shifting } \\
\text { to another position. }\end{array}$ \\
Walk & $\begin{array}{l}\text { Walk is a four-beat gait with the legs following this } \\
\text { sequence: left hind leg, left front leg, right hind leg, } \\
\text { right front leg, leaving three feet on the ground. } \\
\text { Trot is a two-beat diagonal gait where the diagonal } \\
\text { pairs of legs move forward concurrently with a }\end{array}$ \\
& $\begin{array}{l}\text { moment of pause between each beat. } \\
\text { Canter is a three-way gait which begins with the } \\
\text { hind leg, in a rocking motion leads to the front. }\end{array}$ \\
Roll & $\begin{array}{l}\text { The horse starts lying on the ground, rotating } \\
\text { the body over its back, alternating from side to side, } \\
\text { remaining parallel to the performing surface. }\end{array}$ \\
Paw & $\begin{array}{l}\text { The horse scrapes the ground with a forelimb. } \\
\text { The horse looks at its side or flank. } \\
\text { watching }\end{array}$
\end{tabular}

TABLE III

MOVEMENT CLASS 2 S INSTANCES AND PROPORTIONS OF THE STUDIED MOVEMENTS OF ALL HORSES.

\begin{tabular}{llllllll}
\hline & \multicolumn{7}{c}{ Classes } \\
\hline & Stand & Walk & Trot & Canter & Roll & Paw & $\begin{array}{l}\text { Flank } \\
\text { watching }\end{array}$ \\
\hline Horse 1 & 41 & 330 & 129 & 7 & & & \\
Horse 2 & 28 & 201 & 116 & 61 & & & 35 \\
Horse 3 & 10 & 75 & 46 & 10 & & & \\
Horse 4 & 80 & 206 & 155 & 63 & 30 & 20 & \\
Horse 5 & 9 & 165 & 146 & 28 & & & \\
Horse 6 & 29 & 132 & 66 & 18 & & & \\
\hline Instances & 197 & 1111 & 658 & 187 & 30 & 20 & 35 \\
$(\%)$ & 8.8 & 49.6 & 29.4 & 8.4 & 1.3 & 0.9 & 1.6 \\
\hline
\end{tabular}
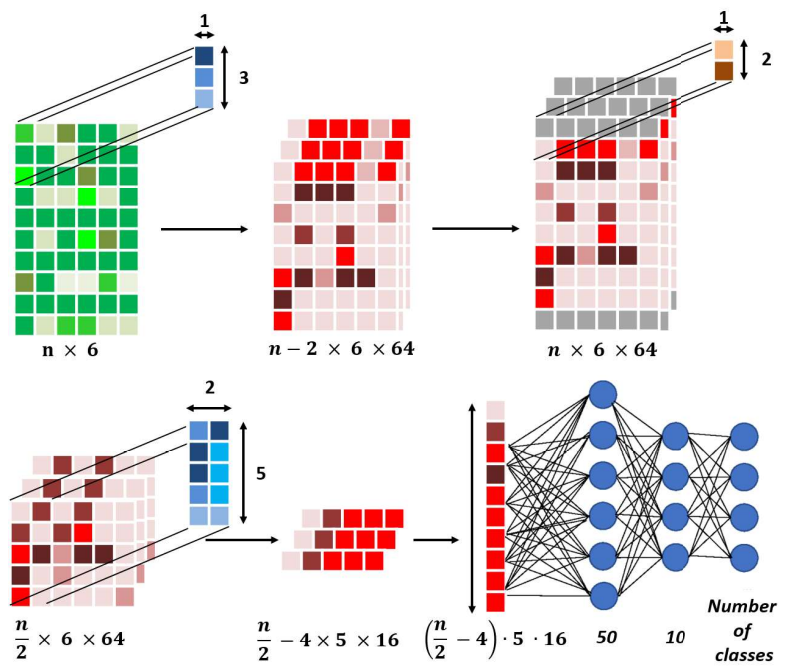

Fig. 2. CNN architecture with $n$ the number of samples as input. 


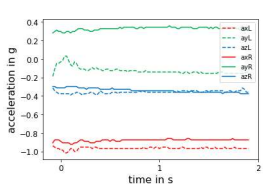

(a) stand

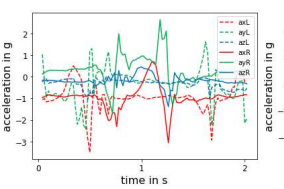

(b) walk

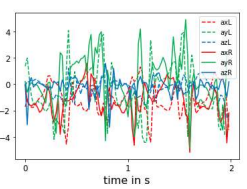

(c) trot

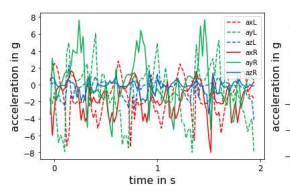

(d) canter

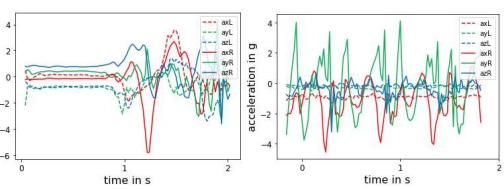

(e) roll

(f) paw

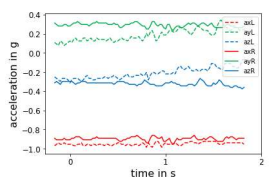

(g) flank watching

Fig. 3. Typical accelerometer patterns of (a) stand, (b) walk, (c) trot, (d) canter, (e) roll, (f) paw and (g) flank watching in a $2 \mathrm{~s}$ window. The red, green, blue dashed lines represent $\mathrm{X}, \mathrm{Y}, \mathrm{Z}$ signals from the left accelerometer and the red, green, blue solid lines represent $\mathrm{X}, \mathrm{Y}, \mathrm{Z}$ signals from the right accelerometer, respectively.

The class proportions of the seven studied activities are not distributed evenly and therefore, in the training phase, the class-wise weights are balanced [18]. Because of this, the cost function penalizes loss more severely on underrepresented classes which can help the model to better adapt to a minority class's characteristics.

The first convolutional layer filters the $n \times 6 \times 1$ input accelerometer data with 64 kernels of size $3 \times 1$ and step size 1 . The L2 regularization technique is used in this layer with a weight decay coefficient of 0.01 [19]. The first convolutional layer is followed by zero-padding such that the output has the same length as the original input. Then a max-pooling operation is performed. The second convolutional layer takes as input the (pooled) output of the first convolutional layer and filters it with 16 kernels of size $5 \times 2$ and stride 1 . Both layers contain an activation layer using rectified units (ReLUs) and dropout of 0.55 is used [7]. The Adam optimizer is used for training the neural network through back propagation.

Training is done for 400 epochs, with an early stopping criterion of halting training when there is no increase in accuracy during the last 60 epochs [20]. The training set is used to train the model, while the validation set is only used to evaluate the model's performance.

Correct selection of the evaluation criteria is crucial for evaluating the merits of a model. In this work, the overall model accuracy, confusion matrices and classification time per sample of validation data are considered for the model performance assessment.

\section{RESULTS}

The deep network in this work is developed and evaluated in Python language using Keras with Tensorflow as backend. The experiments are performed on a Dell inc. with an Intel(R) Core(TM) i7-8650U CPU (1.90GHz) processor.

Figure 3 shows two second data windows of the four gaits and the other behaviours, from the left and right accelerometer worn on the lateral side of the tendon boot. Standing data typically appear as constant signals indicating less movement (figure $3 \mathrm{a}$ ) while faster gait data consist of fluctuating movements (figure $3 b, 3 c$ and $3 d$ ).

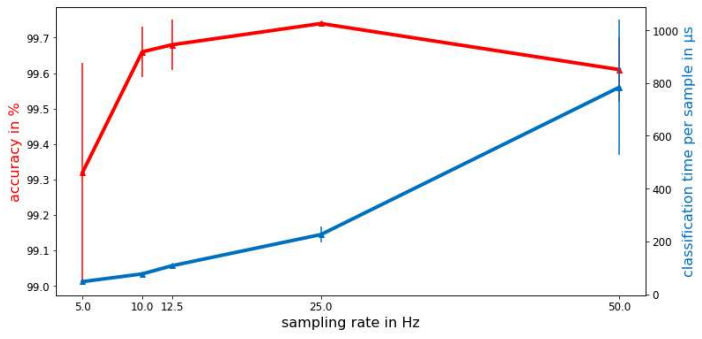

Fig. 4. Mean performance of the convolutional neural network with increasing sampling rate together with the mean classification time per sample.

TABLE IV

MEAN DURATION OF BEHAVIOUR FOR THE PARTICIPATING HORSES.

\begin{tabular}{lllllll}
\hline & Walk & Trot & Canter & Roll & Paw & $\begin{array}{l}\text { Flank } \\
\text { watching }\end{array}$ \\
\hline Mean (s) & 1.23 & 0.77 & 0.58 & 2.21 & 0.54 & 4.87 \\
\hline
\end{tabular}

First, the effect of sampling rate reduction is evaluated. Additionally, measurement noise and rotations along the $\mathrm{X}, \mathrm{Y}$ and $\mathrm{Z}$-axes are added to the training set, validation set and both. Finally, the model is evaluated on different horse types.

\section{A. Resampling}

The first topic which we investigate is the effect of resampling on the classification accuracy. Existing data measured at $50 \mathrm{~Hz}$ was resampled to lower sampling rates i.e., $5 \mathrm{~Hz}, 10 \mathrm{~Hz}, 12.5 \mathrm{~Hz}$, $25 \mathrm{~Hz}$. The training data for the $\mathrm{CNN}$ is obtained by automatically splitting the dataset in two parts: two thirds for training and one third for validation. The mean validation accuracy using ten-foldcross validation together with the mean classification time per sample for each sampling rate are depicted in Figure 4. The vertical lines represent the standard deviation. As can be concluded from this figure the baseline performance of the model improves from an average accuracy of $99.61 \%$ at a sampling rate $50 \mathrm{~Hz}$ to a maximum average accuracy of $99.74 \%$ at a sampling rate of $25 \mathrm{~Hz}$ and the average accuracy of the model decreased with about $0.42 \%$ when the sampling rate was reduced from $25 \mathrm{~Hz}$ to $5 \mathrm{~Hz}$. Figure 5 shows exemplar confusion matrices of the validation data. One misclassification of trot, one misclassification of roll and one or two misclassifications of flank-watching are occuring. The classification time per sample is increasing 16-fold from $5 \mathrm{~Hz}$ to $50 \mathrm{~Hz}$ which is as expected since the input vector size increases from $10 \times 6 \times 1$ to $100 \times 6 \times 1$ and thus computational complexity increases. The classification time is in any case lower than $1100 \mu$ s which is good since the mean duration of the shortest behaviour of all the subjects as is presented in Table IV is 529 times higher than the classification time.

\section{B. Jittering}

The second question was how noise affects the performance of the model. For jittering, a zero-mean Gaussian distribution with a standard deviation value variating from 0.1 to 1 STD in steps of 0.1 is sampled and a Gaussian noise is generated for a time window of $2 \mathrm{~s}$ in the six directions of the $50 \mathrm{~Hz}$ signal for the length of the training data, the validation data and full dataset. Again the training and validation data is splitted automatically with two thirds for training and one third for validation. As can be concluded from the results that are presented in Figure 6, still high accuracies are reached $(\geq 99 \%)$ for three cases when a noise of 0.3 STD is added to the signal. When noise is only added to the validation set a fast decline in accuracy after adding 0.3 STD of noise can be noticed. 


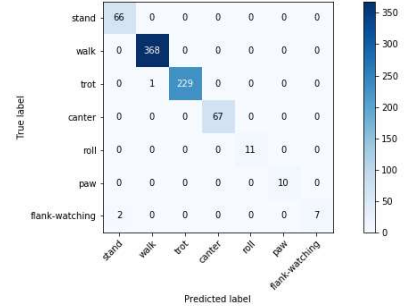

(a) $5 \mathrm{~Hz}, 99.61 \%$

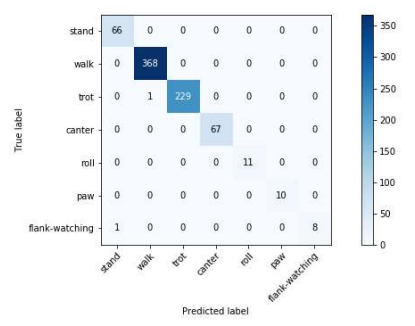

(c) $12.5 \mathrm{~Hz}, 99.74 \%$

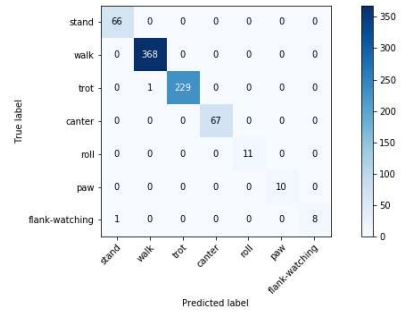

(b) $10 \mathrm{~Hz}, 99.74 \%$

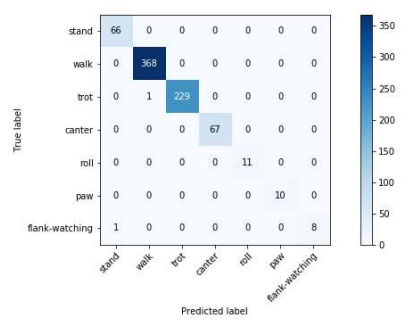

(d) $25 \mathrm{~Hz}, 99.74 \%$

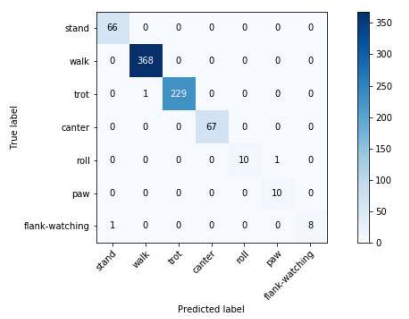

(e) $50 \mathrm{~Hz}, 99.61 \%$

Fig. 5. Exemplar confusion matrices of the validation data for the model validated on accelerometer data with sampling rates of $5 \mathrm{~Hz}(\mathrm{a}), 10 \mathrm{~Hz}$ (b), $12.5 \mathrm{~Hz}(\mathrm{c}), 25 \mathrm{~Hz}$ (d) and $50 \mathrm{~Hz}$ (e)

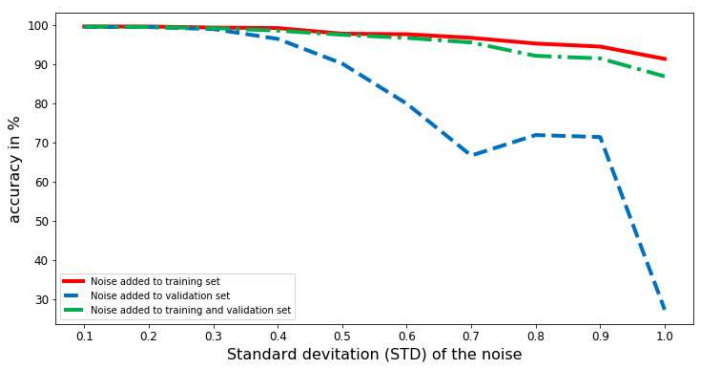

Fig. 6. Mean performance of the convolutional neural network with increasing standard deviation of the noise for noise added to the training set (red), validation set (blue) and both (green).

The baseline accuracy of $99.61 \%$ is in any case never exceeded. The classification time per sample when noise is added to the training set, to the validation set and to both when obtaining results higher than $99 \%$ is in the same order of magnitude compared to when no noise is added i.e., $450 \mu \mathrm{s} \pm 34,851 \mu \mathrm{s} \pm 314,399 \mu \mathrm{s} \pm 26$ vs $784 \mu \mathrm{s} \pm 255$, respectively.

\section{Rotation}

The third topic of investigation was the effect of various sensor placements on the overall validation accuracy. The left and right accelerometer $3 \mathrm{D}$ data are rotated simultaneously along one of the $\mathrm{X}, \mathrm{Y}$ or $\mathrm{Z}$ axes by selecting an angle from $0^{\circ}$ to $180^{\circ}$, building a 3D rotation matrix and applying this transformation to the training data,

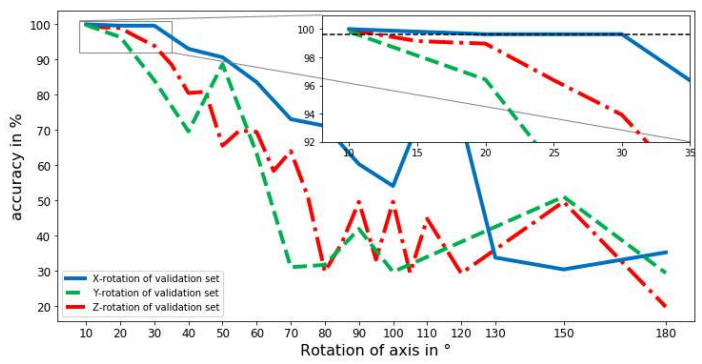

Fig. 7. Mean performance of the convolutional neural network with increasing rotation along the X-axis (blue), Y-axis (green) and Z-axis (red) of left and right accelerometer data for the validation set with the striped line in the zoom box indicating the baseline accuracy of $99.61 \%$.

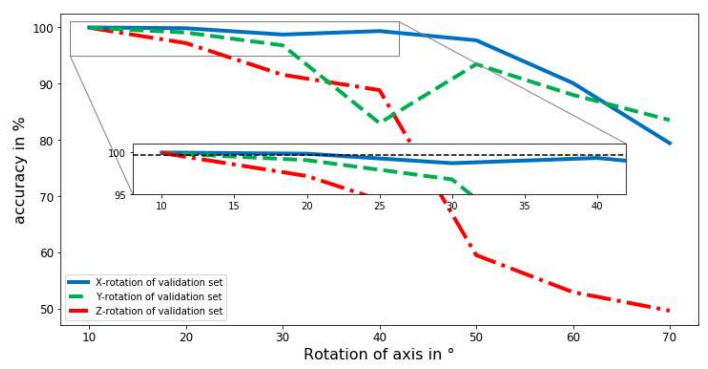

Fig. 8. Mean performance of the convolutional neural network with increasing rotation for rotation added to the $\mathrm{X}$-axis (blue), Y-axis (green) and $\mathrm{Z}$-axis (red) of left and right accelerometer data for the training set with the striped line in the zoom box indicating the baseline accuracy of $99.61 \%$.

validation data and both. Again, a training and test set are obtained by automatically splitting the dataset in two parts: two thirds for training data and one third for validation data. As can be concluded from the results of rotations applied only to the validation set as presented in Figure 7 it is clear that when rotating around the Y-axis, the accuracy decreases faster than when rotating around the $\mathrm{X}$ and $\mathrm{Z}$-axis. But in any case, a rotation of $10^{\circ}$ improves the baseline accuracy from $99.61 \%$ to $100 \%, 99.82 \%$ and $99.96 \%$ for rotations around the $\mathrm{X}, \mathrm{Y}$, and Z-axis, respectively. Rotations around the $\mathrm{Y}$ and $\mathrm{Z}$-axis larger than or equal to $20^{\circ}$ quickly decrease the model accuracy below $99 \%$ but $\mathrm{X}$-axis rotations only have a significant impact from $30^{\circ}$ on. Rotations larger than $30^{\circ}$ decrease in any case the classification accuracy. As can be concluded from the results of rotations applied only to the training set as presented in Figure 8 it is clear that when rotating around the $\mathrm{Z}$-axis, the accuracy decreases faster than when rotating around the $\mathrm{X}$ and $\mathrm{Y}$-axis. But in any case again, a rotation of $10^{\circ}$ improves the baseline accuracy from $99.61 \%$ to $99.91 \%$ for rotations around the $\mathrm{Y}$ and $\mathrm{Z}$-axis and to $99.96 \%$ for a rotation around the $\mathrm{X}$-axis. Rotations around the $\mathrm{Y}$ and $\mathrm{Z}$-axis larger than or equal to $30^{\circ}$ decrease the model accuracy below $99 \%$ but for $\mathrm{X}$-rotations up to $40^{\circ}$ the model accuracy stays around $99 \%$. Rotations larger than $40^{\circ}$ decrease in any case the classification accuracy. The model accuracy increases on average with $0.32 \%$ (99.61\% vs $99.93 \%$ ) when applying a $10^{\circ} \mathrm{X}, \mathrm{Y}$ or $\mathrm{Z}$ rotation to the validation or training set. As can be concluded from the results of the model trained and validated on rotations presented in Table $\mathrm{V}$ the baseline accuracy of $99.61 \%$ is in many cases exceeded with an average of $99.69 \% \pm 0.07$ for all the investigated rotations. This indicates that the generalization ability of the model is improved when applying small $\mathrm{X}, \mathrm{Y}$ or Z-rotations $\left(\leq 10^{\circ}\right)$ to the training or validation set and any $\mathrm{X}, \mathrm{Y}$ or $\mathrm{Z}$-rotations between $0^{\circ}$ and $180^{\circ}$ when the model is trained and validated on these data. The average classification time per sample is $419 \mu$ s when 
TABLE $V$

PERFORMANCE OF THE CNN WITH VARIOUS ROTATIONS OF THE X, Y AND Z-AXIS OF THE LEFT AND RIGHT ACCELEROMETER APPLIED TO THE TRAINING AND TEST SET.

\begin{tabular}{|c|c|c|c|c|c|c|c|c|c|c|c|c|c|}
\hline \multirow[t]{3}{*}{ Rotation $\left(^{\circ}\right)$} & $\mathrm{x}$-axis & 45 & 90 & 135 & 180 & 0 & 0 & 0 & 0 & 0 & 0 & 0 & 0 \\
\hline & $y$-axis & 0 & 0 & 0 & 0 & 45 & 90 & 135 & 180 & 0 & 0 & 0 & 0 \\
\hline & $\mathrm{z}$-axis & 0 & 0 & 0 & 0 & 0 & 0 & 0 & 0 & 45 & 90 & 135 & 180 \\
\hline Accuracy (\%) & & 99.80 & 99.67 & 99.74 & 99.67 & 99.74 & 99.61 & 99.67 & 99.67 & 99.80 & 99.67 & 99.54 & 99.67 \\
\hline
\end{tabular}

obtaining results higher than $99 \%$ for the three cases when a $\mathrm{X}, \mathrm{Y}$ or Z-rotation is added to the training set, to the validation set and to both and is thus in the same order of magnitude than when no rotations are added.

\section{Validation on unseen horse}

The last research question was how the model accuracy was influenced by the type of horse the model was validated on while the model is trained on the other horses to further asses the generalization of the model. To validate the $\mathrm{CNN}$ on unseen data, the datasets are manually splitted into a training set and a validation set. The model is trained on all the datasets available except the data of the validation horse. Furthermore as shown in Table III, all horses performed the four movements i.e., 'stand', 'walk', 'trot' and 'canter' while not every horse performed the other behaviours and solely those are thus investigated.

The confusion matrices of the model validated on six different subjects performing four different movements are depicted in Figure 9 together with the overall model accuracy and mean classification time for each sample. As can be concluded from the confusion matrices, the model validated on Horse 1-2-4-5 reach all accuracies above or equal to the baseline accuracy of $99.61 \%$, while validation of the model on horse 3 and 6 are performing the least. Those results are in the line of expectation since horse 3 is a lame horse and they have asymmetrical gait patterns because they consistently shorten the cranial (forward) phase of stride [21]. The overall validation accuracy is $97.16 \%$ due to the misclassification of 'trot' which gets classified as 'walk' and 'canter'. Horse 6 is a pony and the mean acceleration values per second during the gaits trot and walk are higher for ponies than for horses [4]. Therefore, the model has more difficulties classifying unseen data from a pony. The overall validation accuracy is $95.51 \%$ and the classes that are least performing are 'trot' and 'canter'. In all cases, the classes 'stand' and 'walk' get classified with an accuracy of $100 \%$. The mean classification time is more or less the same in all cases and is around $402 \mu \mathrm{s}$.

\section{CONCLUSION}

In this study we propose a horse activity recognition solution, using Convolutional Neural Networks with wearable sensor accelerometer data as input. It has the benefits of using small sampling rate of $5 \mathrm{~Hz}$ and short classification times per sample up to $48 \mu$ s for obtaining accuracies of $99.31 \%$ and requiring no feature engineering. To evaluate the performance of the considered approach we applied data augmentation techniques such as jittering and rotation and tested it on the training set, validation set and both. The obtained results demonstrate that the proposed CNN-based model achieves high accuracies $(\geq 99 \%)$ when Gaussian noise smaller than 0.3 standard deviation is applied in all the cases. Applying a counter-clockwise $\mathrm{X}$, $\mathrm{Y}$ or Z-rotation of $10^{\circ}$ to the training set or validation set improved the baseline performance in any case and even an accuracy of $100 \%$ was reached when applying a $10^{\circ} \mathrm{X}$-axis rotation to the validation set. Applying rotations to the training and test set did increase the overall classification accuracy on average with $0.08 \%$. A model that was trained on healthy horses, was validated on unseen data of a

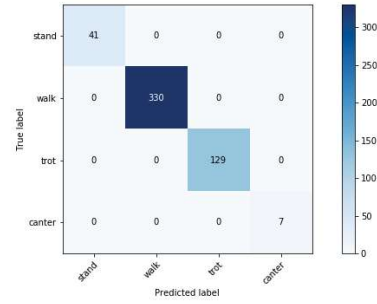

(a) Horse $1,100 \%, 410 \mu \mathrm{s}$

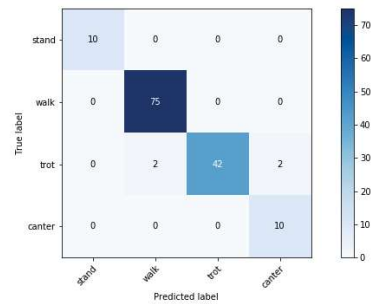

(c) Horse 3, 97.16\%, $401 \mu \mathrm{s}$

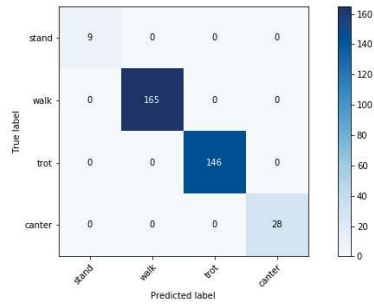

(e) Horse 5, 100\%, $394 \mu \mathrm{s}$

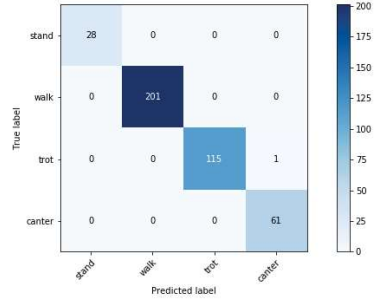

(b) Horse 2, 99.75\%, $378 \mu \mathrm{s}$

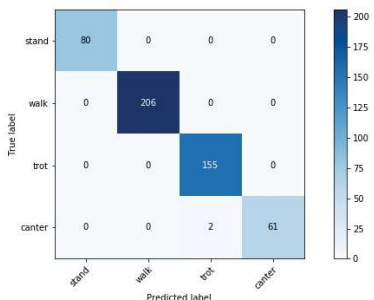

(d) Horse 4, 99.60\%, $439 \mu \mathrm{s}$

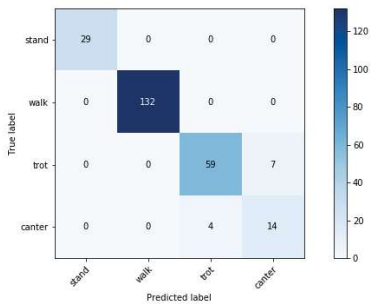

(f) Horse 6, 95.51\%, $392 \mu \mathrm{s}$
Fig. 9. Confusion matrices of the validation data for the model validated on the six different test subjects with the overall model accuracy and mean classification time per sample.

lame horse and a pony resulting in a limited accuracy decrease of no more than $4.49 \%$. The experiment has emphasized that the proposed algorithm can be applied not only to different subjects, but can be used with different measurement disturbances such as noise and sensor rotation.

\section{ACKNOWLEDGMENT}

This work was executed within the imec.icon project Hoof-MATE, aresearch project bringing together academic researchers and industry partners. The Hoof-MATE project was co-financed by imec andreceived project support from Flanders Innovation Entrepreneurship (project nr. HBC.2018.0536).

\section{REFERENCES}

[1] S. Neethirajan, S. K. Tuteja, S.-T. Huang, and D. Kelton, "Recent advancement in biosensors technology for animal and livestock health management," Biosensors and Bioelectronics, vol. 98, pp. 398-407, 2017. 
[2] J. P. van Loon and M. C. Van Dierendonck, "Monitoring acute equine visceral pain with the equine utrecht university scale for composite pain assessment (equus-compass) and the equine utrecht university scale for facial assessment of pain (equus-fap): a scale-construction study," The Veterinary Journal, vol. 206, no. 3, pp. 356-364, 2015.

[3] R. Langrock, R. King, J. Matthiopoulos, L. Thomas, D. Fortin, and J. M. Morales, "Flexible and practical modeling of animal telemetry data: hidden markov models and extensions," Ecology, vol. 93, no. 11, pp. 2336-2342, 2012.

[4] J.-B. Burla, A. Ostertag, H. S. Westerath, and E. Hillmann, "Gait determination and activity measurement in horses using an accelerometer," Computers and electronics in agriculture, vol. 102, pp. 127-133, 2014.

[5] O. R. Bidder, H. A. Campbell, A. Gómez-Laich, P. Urgé, J. Walker, Y. Cai, L. Gao, F. Quintana, and R. P. Wilson, "Love thy neighbour: automatic animal behavioural classification of acceleration data using the k-nearest neighbour algorithm,' PloS one, vol. 9, no. 2, p. e88609, 2014.

[6] S. Benaissa, F. A. Tuyttens, D. Plets, T. de Pessemier, J. Trogh, E. Tanghe, L. Martens, L. Vandaele, A. Van Nuffel, W. Joseph et al., "On the use of on-cow accelerometers for the classification of behaviours in dairy barns," Research in veterinary science, 2017.

[7] N. Srivastava, G. Hinton, A. Krizhevsky, I. Sutskever, and R. Salakhutdinov, "Dropout: a simple way to prevent neural networks from overfitting," The Journal of Machine Learning Research, vol. 15, no. 1, pp. 1929-1958, 2014.

[8] X. Zhao, H. Wei, H. Wang, T. Zhu, and K. Zhang, "3d-cnn-based feature extraction of ground-based cloud images for direct normal irradiance prediction," Solar Energy, vol. 181, pp. 510-518, 2019.

[9] D. Ravi, C. Wong, B. Lo, and G.-Z. Yang, "A deep learning approach to on-node sensor data analytics for mobile or wearable devices," IEEE journal of biomedical and health informatics, vol. 21, no. 1, pp. 56-64, 2017.

[10] S. Bersch, D. Azzi, R. Khusainov, I. Achumba, and J. Ries, "Sensor data acquisition and processing parameters for human activity classification," Sensors, vol. 14, no. 3, pp. 4239-4270, 2014.

[11] K. Cobbe, O. Klimov, C. Hesse, T. Kim, and J. Schulman, "Quantifying generalization in reinforcement learning," arXiv preprint arXiv:1812.02341, 2018.

[12] J. Munoz-Bulnes, C. Fernandez, I. Parra, D. Fernández-Llorca, and M. A. Sotelo, "Deep fully convolutional networks with random data augmentation for enhanced generalization in road detection," in 2017 IEEE 20th International Conference on Intelligent Transportation Systems (ITSC). IEEE, 2017, pp. 366-371.

[13] V. Varkarakis, S. Bazrafkan, and P. Corcoran, "Deep neural network and data augmentation methodology for off-axis iris segmentation in wearable headsets," Neural Networks, vol. 121, pp. 101-121, 2020.

[14] T. T. Um, F. M. J. Pfister, D. Pichler, S. Endo, M. Lang, S. Hirche, U. Fietzek, and D. Kulić, "Data augmentation of wearable sensor data for parkinson's disease monitoring using convolutional neural networks," arXiv preprint arXiv: 1706.00527, 2017.

[15] R. Reed and R. J. MarksII, Neural smithing: supervised learning in feedforward artificial neural networks. Mit Press, 1999.

[16] H. Brugman, A. Russel, and X. Nijmegen, "Annotating multimedia/multi-modal resources with elan." in LREC, 2004.

[17] G. A. Sutton, R. Dahan, D. Turner, and O. Paltiel, "A behaviour-based pain scale for horses with acute colic: scale construction," The Veterinary Journal, vol. 196, no. 3, pp. 394-401, 2013.

[18] S. Li, W. Song, H. Qin, and A. Hao, "Deep variance network: An iterative, improved cnn framework for unbalanced training datasets," Pattern Recognition, vol. 81, pp. 294-308, 2018.

[19] A. A. Mallouh, Z. Qawaqneh, and B. D. Barkana, "Utilizing cnns and transfer learning of pre-trained models for age range classification from unconstrained face images," Image and Vision Computing, 2019.

[20] C. A. Ronao and S.-B. Cho, "Human activity recognition with smartphone sensors using deep learning neural networks," Expert systems with applications, vol. 59, pp. 235-244, 2016.

[21] E. J. Davidson, "Lameness evaluation of the athletic horse," Veterinary Clinics: Equine Practice, vol. 34, no. 2, pp. 181-191, 2018. 